\title{
SIMULAÇÃO HIL (HARDWARE IN THE LOOP) DE UM MOTOR COM TAXA DE COMPRESSÃO VARIÁVEL
}

\author{
André Vinicius Oliveira Maggio ${ }^{1}$, Armando Antônio M. Laganá ${ }^{1}$ e Joao Francisco Justo \\ Filho $^{1}$ \\ ${ }^{1}$ Escola Politécnica da Universidade de São Paulo
}

E-mails: andremaggio@usp.br, lagana@1si.usp.br, justo@1me.usp.br.

\section{RESUMO}

Nos últimos anos, a demanda por motores mais eficientes que trabalhem com mais de um tipo de combustível (Flex) tem crescido no mercado. Um dos fatores que influenciam diretamente a eficiência é a sua taxa de compressão, embora os veículos atuais ainda são equipados com motores com taxa fixa. Recentemente, foi anunciado o primeiro veículo equipado com taxa de compressão variável (VCR), tecnologia que tem sua importância aumentada significativamente para motores do tipo Flex. Visando o desenvolvimento de estratégias de para controle da taxa de compressão e, considerando a dificuldade envolvida na construção ou a obtenção de um motor equipado com essa tecnologia, uma possível solução é a elaboração de uma simulação por Hardware-in-the-loop (HIL) implementando um motor com taxa de compressão variável virtual a partir dos dados coletados em ensaios num motor convencional de taxa fixa, porém, adaptado com cabeçotes e pistões diferentes dos originais, a fim de coletar dados para uma taxa diferente do original, permitindo entender a dinâmica do motor em função da variação da taxa de compressão.

\section{INTRODUÇÃO}

O tipo mais comum de motor que equipa a maioria dos veículos é o de ciclo Otto. Suas características principais são a ignição por centelha e um ciclo de quatro tempos. A taxa de compressão $(\varepsilon)$ é uma das propriedades geométricas do motor, sendo fixa para um motor convencional e otimizada dependendo da finalidade do motor, como, por exemplo, desempenho ou economia, e do tipo de combustível utilizado.

A taxa de compressão é definida como a relação entre os volumes do cilindro no ponto morto inferior e no ponto morto superior [1].

$b \quad$ é o diâmetro do pistão.

$$
\varepsilon=\frac{\frac{\pi}{4} \times b^{2} \times S+V_{c}}{V_{r}}
$$

$S \quad$ é o curso do pistão.

$V_{c} \quad$ é o volume da câmara de combustão. 
Cada tipo de combustível tem uma pressão limite durante o ciclo de compressão para um dado motor com específicas condições iniciais de temperatura e rotação no ciclo estudado. Exceder esta pressão pode levar à autoignição da mistura ar combustível, em que, ao invés de uma combustão normal desejável, gera outro fenômeno dentro da câmara de combustão: a detonação $(k n o c k)$.

Em uma combustão normal, a frente de chama começa a partir da centelha produzida pela vela de ignição e percorre a câmara de combustão liberando energia, elevando a temperatura e, consequentemente, a pressão dentro do cilindro, produzindo trabalho útil no ciclo de expansão. A detonação é uma ignição espontânea de pelo menos parte da mistura ar/combustível, formando uma onda de choque ao invés da frente de chama suave da combustão. Esta onda de choque eleva a pressão e a temperatura no cilindro, podendo gerar simultaneamente outra detonação no cilindro. Isto reduz o rendimento térmico do motor e, dependendo da intensidade e frequência com que ocorre, pode diminuir a vida útil do motor.

A operação do motor em regime de carga elevada demanda maior volume de mistura ar/combustível para suprir a demanda de potência. Isto gera um aumento na pressão durante a compressão, que pode resultar na detonação da mistura. Normalmente, a ECU controla o ponto de ignição, a fim de evitar a detonação às custas de uma perda no desempenho térmico do motor. Outra possibilidade seria reduzir a taxa de compressão, aumentando a potência, e consequentemente, o desempenho do motor quando opera em regime de carga elevado. Entretanto, quando o motor é submetido a um regime de carga mais leve, o motor perde eficiência devido à baixa taxa de compressão, elevando o consumo de combustível. A solução definitiva para esse problema seria a possibilidade de ajustar a taxa de compressão, tecnologia que, assim como às demais tecnologias de injeção direita de combustível e turbo compressores, promete aumento de rendimento, economia de combustível e o uso de diversos tipos de combustíveis além da gasolina, como o GPL e o etanol.

A influência da taxa no rendimento obedece uma relação que pode ser vista em (2), relação que resulta da termodinâmica [1]. Já a do ponto de ignição obedece a uma relação do tipo quadrática [1]. Para regimes de alta demanda de potência e desempenho, adotar ângulos de ignição muito diferentes do ótimo prejudica de forma agressiva a potência (2). Por outro lado, abaixar a taxa de compressão permite obter potências maiores devido ao ligeiro aumento da capacidade volumétrica do cilindro. É claro que existe uma estratégia ótima de avanço de ignição e redução da taxa para cada tipo de regime em que o motor é submetido, porém um motor equipado com a tecnologia VCR (Variable Compression Ratio) tem um desempenho e respostas superiores.

$$
\begin{gathered}
e(\zeta)=1-k_{\zeta} \cdot\left(\zeta-\zeta_{0}\left(\omega_{\theta}, p_{m e}\right)\right) \\
e(\varepsilon)=\frac{1-1 / \varepsilon^{k_{\varepsilon}}}{1-1 / \varepsilon_{0} k_{\varepsilon}}
\end{gathered}
$$

e é a eficiência relativa, que multiplica a eficiência global do motor que depende de diversos fatores. Seu valor vai de zero a um, no qual se tem o rendimento global ótimo do motor.

$\varepsilon_{0} \quad$ é a taxa de compressão máxima do motor. 
$\varepsilon \quad$ é a taxa de compressão do motor. Quando seu valor é igual a $\varepsilon_{0}, \mathrm{e}=1$.

$\mathrm{k}_{\varepsilon} \quad$ é uma constante que depende do coeficiente de expansão adiabática da temperatura e composição dos gases. Seu valor típico é 0,55.

$\zeta \quad$ é o angulo de ignição.

$\zeta_{0} \quad$ é o ângulo ótimo de ignição. Seu valor depende da rotação $\omega_{\mathrm{e}} \mathrm{e}$ do regime de carga

pme.

$\mathrm{k}_{\zeta} \quad$ é um coeficiente experimental.

\section{TAXA DE COMPRESSÃO VARIÁVEL (VCR)}

A tecnologia VCR permite ajustar a taxa de compressão de um motor durante as diversas condições de operação. Esta tecnologia já existia há algum tempo em laboratórios de desenvolvimento, porém somente agora está se tornando viável em escala industrial, tanto que recentemente foi anunciado o primeiro veículo equipado com essa tecnologia.

Conforme a equação (1), a taxa é função do volume da câmara de combustão $\left(\mathrm{V}_{\mathrm{c}}\right)$. A principal estratégia dos motores de compressão variável é permitir que o volume da câmara de combustão possa ser alterado. Isto pode ser feito de duas formas: Uma delas seria fazer uso de um sistema mecânico conectado a um atuador que permita variar a distância entre o pistão e o cabeçote do motor. Outra alternativa consiste em introduzir um volume no cabeçote de forma a reduzir o volume da câmara de combustão, o que pode ser feito com um segundo pistão menor no topo do cabeçote, conforme a figura 1.
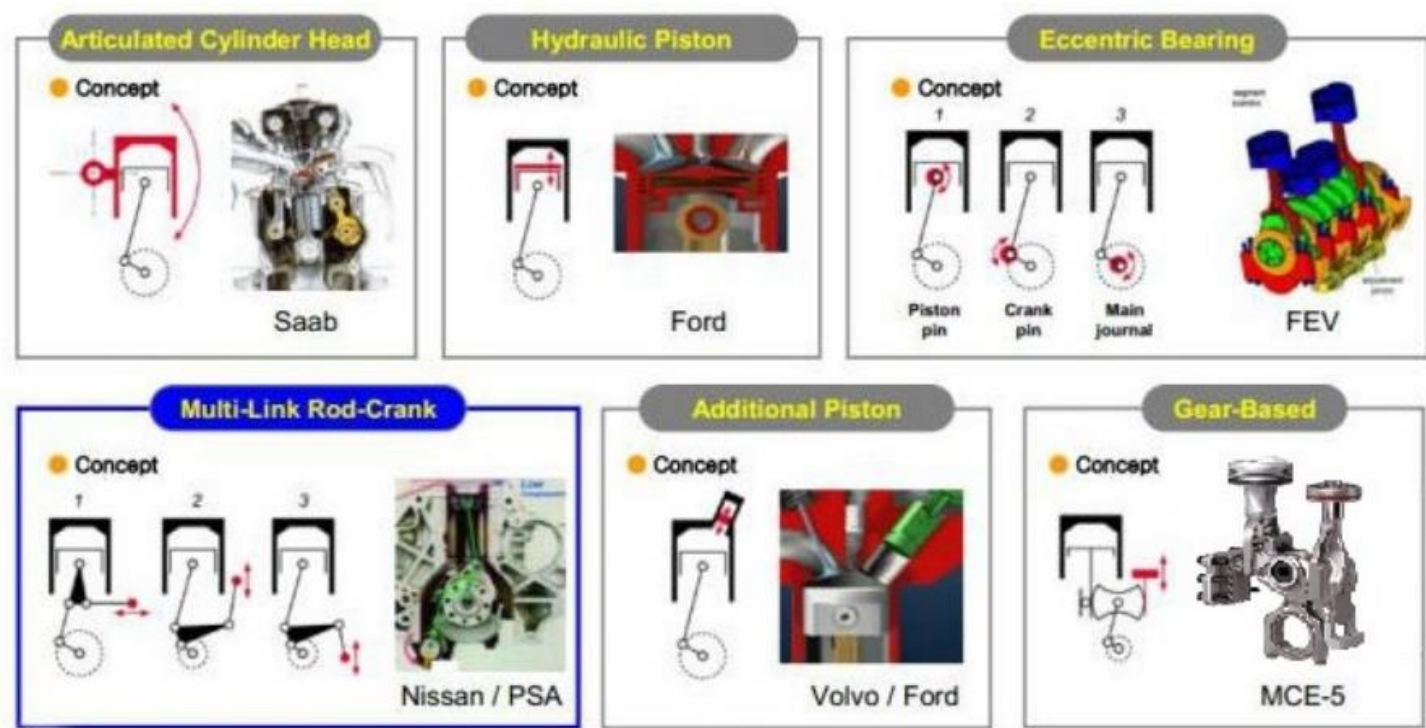

Figura 1: Diferentes mecanismos que permitem variar a taxa de compressão. Fonte [2]

\section{SIMULAÇÃO HIL}

A técnica de simulação hardware-em-loop (HIL) é amplamente utilizada no desenvolvimento e teste de sistemas complexos que demandam simulações em tempo real. Em um sistema de desenvolvimento automotivo antigo, usa-se um motor de teste que permite certos ajustes em sua dinâmica e é equipado com diversos sensores, sendo este motor de custo mais elevado. Deste modo, cada componente na etapa de desenvolvimento pode ser alterado. 
Para o desenvolvimento do software de controle da ECU, fazer uso de um motor real nas primeiras etapas de desenvolvimento não é muito interessante. Nesse caso, uma simulação HIL seria recomendada. Inicialmente, é feita uma primeira etapa de desenvolvimento em um ambiente puramente virtual (SIL, software in the loop). A seguir, o modelo de controle é inserido no hardware físico da ECU e é feita uma simulação do processo em outro hardware especifico, que realiza uma simulação de alta confiabilidade em tempo real do motor estipulado no projeto do veículo (figura 2). Então, resta apenas a calibração final da ECU no motor real. Com esse procedimento, ganha-se em termos de custos e tempo de desenvolvimento.
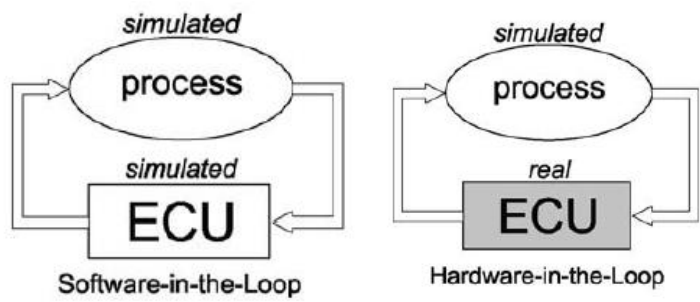

Figura 2: Software in the loop e hardware in the loop. Fonte [3]

Conforme a figura 3, a simulação por HIL inclui a emulação de diversos sensores e atuadores presentes no motor (interface system). Esse bloco interage diretamente com o modelo de simulação da planta, no caso o motor (engine model). É lido pelo hardware em teste, no caso a ECU, que roda os algoritmos de controle (control model). Então, toda a complexidade e custo envolvido em manter uma planta de teste é substituído por uma representação matemática de todos os sistemas dinâmicos. Esse hardware pode ser aproveitado para emular um grande conjunto de motores e sensores bastando apenas sua reprogramação.

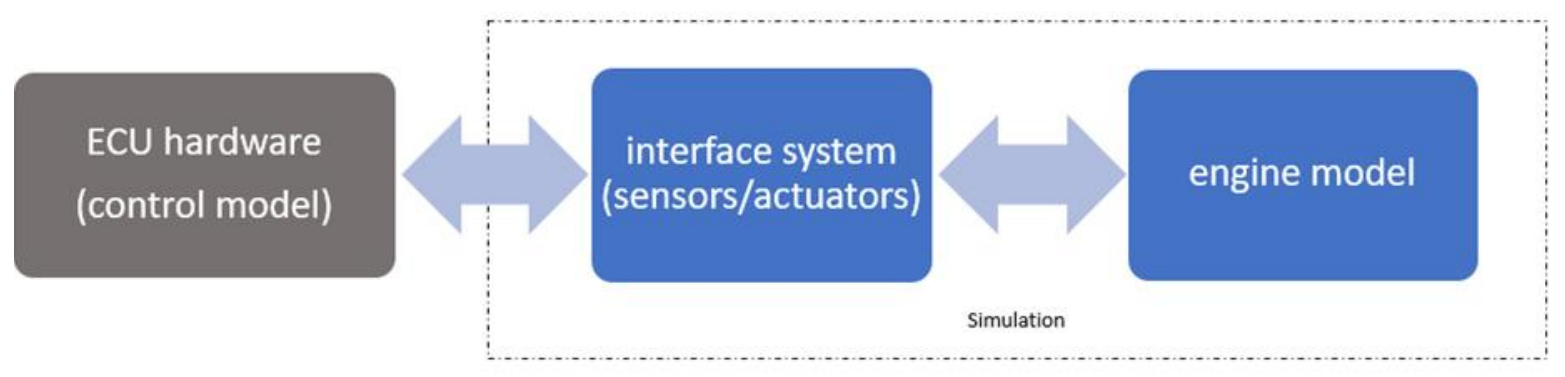

Figura 3: Sistema HIL típico. Fonte: Autor

O uso de HIL melhora a qualidade e profundidade do teste, uma vez que a planta real impõe certas limitações na intensidade e condições dos testes, além da verificação das condições de falha em tempo real. Testar uma unidade de controle do motor com uma planta real pode criar, por exemplo, condições perigosas que diminuiriam muito a vida útil de um motor de teste ou colocaria em risco a segurança de terceiros.

\section{O MOTOR FLEX}

Outra vantagem na utilização de motores VCR pode ser observada no sistema FLEX, uma vez que cada combustível requer uma taxa de compressão adequada. Por exemplo, os motores a etanol podem trabalhar com uma taxa de compressão mais elevada (12:1 a 15:1, podendo 
chegar a 16:1) gerando um rendimento maior que um mesmo motor a gasolina, compensando em parte o seu menor poder calorífico (24 MJ/l) em relação a gasolina (34,6 MJ/1).

Utilizar a taxa de compressão com valores idênticos ao dos motores a gasolina (8:1 a 10:1) para o motor FLEX resultaria em um motor com rendimento muito pobre para o etanol, dado o rendimento que poderia ser obtido com uma taxa maior. Já em contrapartida, utilizar a taxa de compressão do etanol seria possível utilizando o atraso do ponto de ignição para evitar a detonação quando o motor opera com gasolina. Porém, isso não permitiria o máximo aproveitamento do trabalho de expansão dos gases gerados com a queima da gasolina, o que reduz muito o rendimento do motor para a gasolina.

Algumas práticas adotadas para resolver este problema, e que são utilizadas nos veículos brasileiros, foi utilizar uma taxa intermediária entre a ideal para o etanol e para a gasolina, com valores típicos entre 10:1 a 12:1, e adição de etanol à gasolina como aditivo para aumentar a octanagem da gasolina às custas de uma perda no seu poder calórico e a inclusão do sensor de detonação e controle de lambda que, apesar de não ser uma tecnologia exclusiva para motores FLEX, é utilizada para identificar a mistura ar/combustível adequada para cada tipo de combustível. Isto é feito através da relação de lambda, para otimizar o ponto de ignição trabalhando no limite do ângulo para evitar a detonação, que compromete a vida útil do motor. O motor com taxa de compressão variável seria a solução definitiva para o sistema FLEX, permitindo obter melhor desempenho para cada tipo de combustível ou mesmo misturas de combustíveis e utilizar ainda combustíveis como GLP e GNV.

\section{IMPLEMENTAÇÃO DE UM MODELO HIL PARA A TECNOLOGIA VCR}

Para gerar um modelo de simulação HIL, a proposta é não alterar o modelo original do motor, mas sim acrescentar um bloco adicional que trabalha em paralelo com o modelo e corrige de forma dinâmica as variáveis de saída do modelo do motor (figura 4). Para tanto, é necessário realizar ensaios com um jogo de cabeçotes e pistões diferentes dos originais a fim de coletar dados para uma taxa diferente do original. Vários veículos produzidos no Brasil são exportados para países vizinhos onde se utiliza gasolina pura (E0). Para tanto, a taxa dos motores utilizados nesses veículos é mais baixa que a dos veículos brasileiros, porém o motor é idêntico, somente os pistões diferem. Adquirindo um jogo de pistões e utilizando cabeçotes rebaixados é possível realizar ensaios tanto para taxas mais baixas do que a original como para taxas mais elevadas.

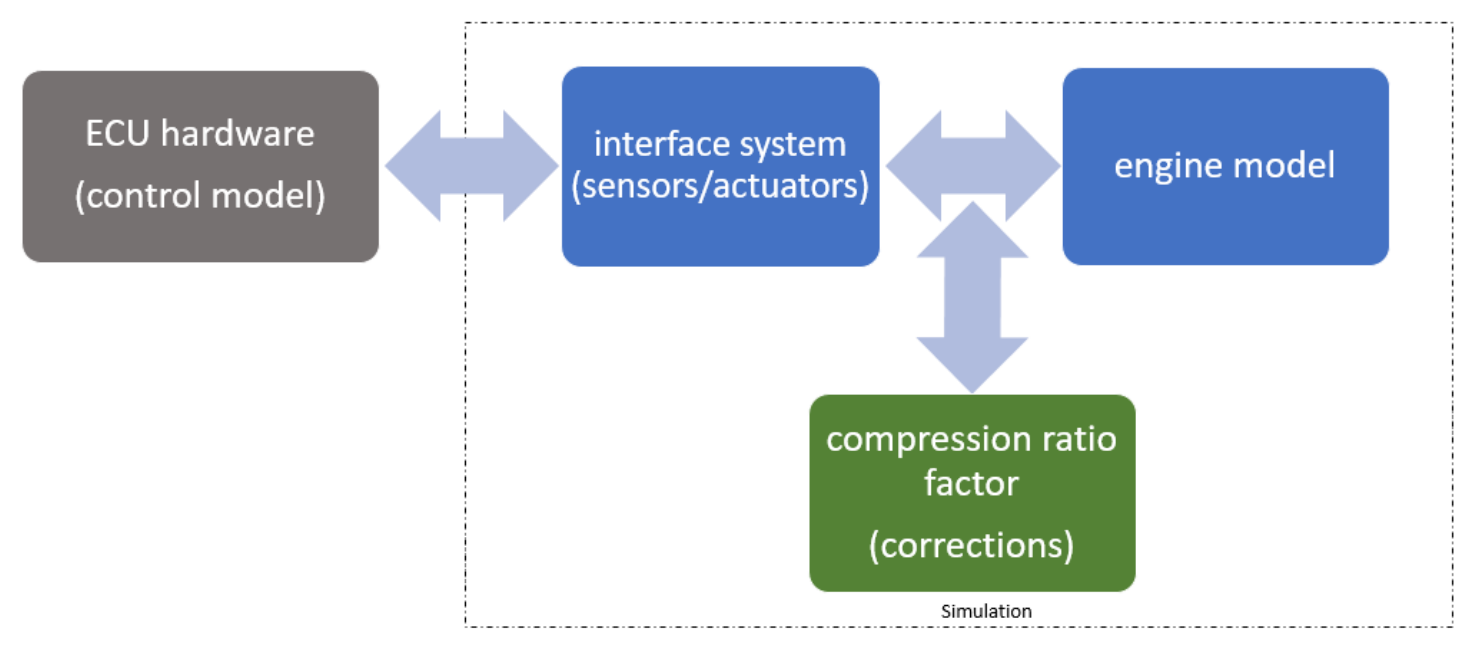


Figura 4: Sistema HIL incorporando o efeito da taxa de compressão variável. Fonte: Autor

Esta seria uma opção de ensaios viáveis para modelagem, tendo em vista a complexidade envolvida em recalcular de forma dinâmica em tempo real todos os parâmetros envolvidos no modelo original do motor e permite, também, tratá-lo como um modelo tipo "caixa preta". Em outras palavras, de forma a não se alterar o modelo já validado para taxa fixa, pode-se criar um bloco a parte, de forma a facilitar a inclusão da compressão variável. A partir das referências [1] e [5], é possível observar que a produção de torque (devido à influência da taxa no rendimento termodinâmico do motor), fator lambda e o knock são diretamente influenciadas pela taxa de compressão.

A ocorrência de knock depende de diversos fatores conforme equação (4) [6].

$$
K_{n}=K_{1} \times\left(1-x_{b} \times \max (1, \phi)\right) \times\left(C R_{0}-1\right) \cdot \sqrt{1-\frac{\theta_{\text {knook }}}{K_{2}}} \times N \cdot \frac{(C R-1)}{\left(C R_{0}-1\right)}
$$

$\mathrm{K}_{\mathrm{n}} \quad$ é um valor experimental relacionado à intensidade do knock.

$\mathrm{K}_{1}$ e $\mathrm{K}_{2}$ são coeficientes experimentais.

$\mathrm{x}_{\mathrm{b}} \quad$ é a fração da massa de combustível queimada

$\phi \quad$ é a razão equivalente combustível ar.

CR é a taxa de compressão.

CR0 é a taxa de compressão original do motor ou referência, sendo seu valor constante.

$\theta_{\text {knock }}$ ângulo do virabrequim no qual o knock inicia.

$\mathrm{N} \quad$ velocidade angular do motor.

Partindo do princípio que a mudança na taxa de compressão do motor só influi no valor de CR e não altera os demais coeficientes da equação, é possivel obter a equação (5), onde a intensidade do knock gerada pelo bloco "engine model" pode ter seu valor corrigido em função da taxa.

$$
K_{n}=K_{n 0} * \frac{(C R-1)}{\left(C R_{0}-1\right)}
$$

CR é a taxa de compressão do motor.

$\mathrm{CR}_{0}$ é a taxa de compressão original do motor ou referência, sendo seu valor constante.

$\mathrm{K}_{\mathrm{n} 0} \quad \mathrm{~K}_{\mathrm{n}}$ considerando o valor de $\mathrm{CR}_{0}$.

Apesar do valor de $\theta_{\text {knock }}$ sofrer alterações, tendo em vista que mudanças na taxa de compressão podem gerar eventuais variações curso do pistão, dependendo do tipo de atuador utilizado para fazê-lo, o valor de $\theta_{\text {knock }} / K_{2}$ é aproximadamente constante, uma vez que as variações curso do pistão são pequenas, da ordem de milímetros, quando comparado ao seu curso total. Da mesma forma, é possível utilizar coeficientes de correção para o fator lambda e para o torque. Para modelar o torque, pode-se analisar a influência da taxa de compressão variável na eficiência, a partir de ensaios realizados em [7].

Estudos que modelam as perdas por fricção das partes mecânicas, como o presente em [8], sugerem que tais perdas dependem apenas do diâmetro e o curso do pistão. Como não há mudança no diâmetro do pistão e como eventuais variações em seu curso conforme modifica- 
se a taxa de compressão podem ser desprezadas, pode-se concluir que as perdas por fricção não variam com mudança da taxa de compressão. Estes conceitos podem ser descritos pela equação:

$$
e_{V C R}(\cdot)=e_{\omega_{s}}\left(\omega_{\theta}, Y\right) \times e_{\lambda}(\lambda, Y) \cdot e_{\zeta}(\zeta, Y) \cdot e_{e g r}\left(x_{e g r}\right) \times e_{\varepsilon}(\varepsilon)
$$

$e_{V C R}(\cdot)$ é a eficiência térmica;

$\omega_{e} \quad$ é a velocidade angular do motor;

$e_{\omega_{\theta}}\left(\omega_{\theta}, \Upsilon\right)$ é o fator da eficiência térmica dependente da velocidade do motor;

$e_{\lambda}(\lambda, Y)$ é o fator da eficiência térmica dependente da relação equivalente ar combustível;

$\zeta$ é o ângulo de ignição;

$e_{\zeta}(\zeta, Y)$ é o fator da eficiência térmica dependente do ângulo de ignição;

$x_{\text {egr }} \quad$ é a taxa de recirculação de gases (pode ser mássica ou molar);

$e_{\text {egr }}\left(x_{\text {egr }}\right)$ é o fator da eficiência térmica dependente da taxa de recirculação de gases.

$e_{s}(\varepsilon)$ é o fator da eficiência térmica dependente da taxa de compressão variável.

$\mathrm{O}$ termo $Y$ se refere a um fator normalizado (que vai de 0 à 1 ), variando linearmente com a composição. Para composições de gasolina tipo A com etanol anidro (escala EX) será usado $Y=0$ para gasolina tipo A e $Y=1$ para etanol anidro. Para composições de gasolina tipo $C$ (E27) com etanol hidratado (escala HX, vide [9]), será usado $Y=0$ para gasolina tipo $\mathrm{C}$ e $\Upsilon=1$ para etanol hidratado.

Um entendimento aprofundado da equação (6), o que não é o foco desta investigação, pode ser encontrado na referência [1]. Através de (6), o torque pode ser modelado como:

$$
\begin{aligned}
& T_{\theta}=e_{\omega_{s}}\left(\omega_{\theta}, Y\right) \times e_{\lambda}(\lambda, Y) \times e_{\zeta}(\zeta, Y) \times e_{\text {egr }}\left(x_{e g r}\right) \times e_{\varepsilon}(\varepsilon) \times m_{\varphi} \times H_{l} \\
& T_{e}=\left(e_{\omega_{s}}\left(\omega_{\theta}, Y\right) \cdot e_{\lambda}(\lambda, \Upsilon) \times e_{\zeta}(\zeta, Y) \times e_{\text {egr }}\left(x_{\text {egr }}\right) \times m_{\varphi} \times H_{l}\right) \times e_{\varepsilon}(\varepsilon)
\end{aligned}
$$

$T_{e} \quad$ é o valor médio de torque produzido em um ciclo determinado;

$m_{\varphi} \quad$ é o valor mássico da mistura de combustível admitida pelo cilindro em um ciclo determinado;

$H_{l} \quad$ é o poder calorífico inferior da mistura de combustível utilizada.

Acima, observa-se a representação qualitativa da abordagem de controle proposta, representando a influência da taxa de compressão variada no torque com um fator de correção função da citada taxa. No equacionamento exposto, não estão ilustradas as perdas devido à fricção das partes mecânicas e à fricção do fluxo gasoso. Porém, ao utilizar um método de identificação apropriado, objetivando aproximar a curva modelada da curva real, o fator $e_{s}(\varepsilon)$ acabará por computar não somente a influência da taxa de compressão, mas também as fricções.

Considerando o sensor lambda, ao variar a taxa de compressão, aumenta-se o volume da câmara de combustão, o que proporciona a admissão de uma quantidade maior de mistura ar/combustível. Porém, sem correções no tempo de injeção, ter-se-á um aumento do valor da razão equivalente ar combustível devido a maior admissão de ar sem a consequentemente maior admissão de combustível. Isso gera um ligeiro empobrecimento ou enriquecimento da 
mistura conforme se varia o valor da taxa de compressão. Esta correção pode ser feita usando a equação (1), calculando-se a porcentagem de excesso ou falta de ar na mistura.

Outros parâmetros que influem na resposta lambda e estão relacionados com o sistema de exaustão são a proporção de gases residuais que permanecem no cilindro, as constantes de tempo da admissão dos gases (do inglês: gas mixing), o atraso de transporte gasoso e a constante de tempo da resposta lambda [1] e [10].

O uso de uma dinâmica de segundo grau para os fenômenos de admissão gasosos dispensa a modelagem da dinâmica dos gases residuais, e é suficiente que a admissão, neste contexto, seja modelada como uma função de transferência constante (que não varia com condições de operação diversas) [1]. Logo, recomenda-se o uso desta abordagem para a admissão, não sendo a modelagem deste fenômeno, portanto, a ser função da compressão variável. A resposta da sonda lambda é uma constante a ser fornecida pelo fabricante.

O atraso de transporte, variante principalmente com a força de bombeamento dos gases de escape, não sofrerá alteração apenas pela mudança isolada da taxa de compressão, pois esta não altera o impulso provocado pelo pistão aos gases de escape. Este impulso é função principalmente da carga e da velocidade do motor, sofrendo pouca modificação com eventuais mudanças no volume da câmara de combustão decorridos da taxa variável.

Com base nessas considerações, observa-se que as dinâmicas dos fenômenos que ocorrem no sistema de exaustão podem ter sua influência advinda da taxa de compressão variável desprezada, bastando que se corrija o tempo de injeção a partir de um fator de correção função unicamente da taxa de compressão. Portanto, o uso de tais fatores de correção é suficiente tanto para o torque quanto no pertinente à resposta da sonda lambda.

\section{CONCLUSÃO}

A simulação HIL é de extrema importância. Reduz custos de projeto e permite ter um motor equipado com a tecnologia VCR mesmo que este seja virtual. Isto permite o desenvolvimento tanto do hardware quanto de estratégias de controle, sem a necessidade de se ter um motor equipado com a tecnologia VCR, ajudando a melhorar as estratégias e a corrigir erros no projeto da ECU que poderiam levar a destruição do motor de teste. Permite também pesquisas de consumo e desempenho do motor, inclusive para a tecnologia FLEX, e comparar de forma qualitativa e quantitativa vantagens e desvantagens de cada atuador empregado para variar a taxa.

A proposta de um sistema que trabalha em paralelo com o modelo original é interessante, uma vez que não se altera o modelo já validado de simulação com taxa fixa. Tem-se, portanto, uma solução flexível que é aplicável com uma facilidade maior a modelos de taxa fixa já em utilização, uma vez que podem ser tratados como modelos tipo "caixa preta". A adição do bloco compression ratio factor na simulação introduz apenas correções nas variáveis a partir dos resultados gerados pelo modelo original. Isto também simplifica a mudança do tipo de atuador que depende da tecnologia VCR empregada (Figura 1), uma vez que pode-se substituir a dinâmica do atuador no bloco de correção sem a necessidade de realizar todos os testes novamente, proporcionando um modelo mais robusto. 


\section{REFERÊNCIAS}

[1] GUZZELLA, Lino; ONDER, Christopher. Introduction to modeling and control of internal combustion engine systems. 2. ed. Springer Science \& Business Media, 2010.

[2] Committee on the Assessment of Technologies for Improving Fuel Economy of LightDuty Vehicles. Cost, Effectiveness and Deployment of Fuel Economy Technologies for Light-Duty Vehicles. 2015. Disponível em https://www.autonews.com/assets/PDF /CA100105618.PDF Acesso em: maio de 2017.

[3] ISERMANN Rolf, MULLER Norbert; Design of computer controlled combustion engines. Article in Mechatronics 13(10):1067-1089, December 2003 with

DOI: $10.1016 / \mathrm{S} 0957-4158(03) 00043-6$

[4] SMITH, P., Heywood, J., and Cheng, W., "Effects of Compression Ratio on Spark-Ignited Engine Efficiency," SAE Technical Paper 2014-01-2599, 2014, doi:10.4271/2014-01-2599

[5] NILSSON Ylva. Modelling for Fuel Optimal Control of a Variable Compression Engine. 2007. Disponível em http://liu.diva-portal.org/smash/get/diva2:24053/ FULLTEXT01.pdf. Acesso em: maio de 2017.

[6] STEURS KAREL F. H. M. Cicle-resolved analysis and modeling of knock in a homogeneous charge spark ignition engine fueled by etanol and iso-octane. 2014. Disponível em https://www.research-collection.ethz.ch/bitstream/handle/20.500.11850/ 154797/eth-47003-02.pdf? sequence=2\&isAllowed=y

[7] SHELBY, M., Leone, T., Byrd, K., and Wong, F., "Fuel Economy Potential of Variable Compression Ratio for Light Duty Vehicles," SAE Int. J. Engines 10(3):2017, doi:10.4271/2017-01-0639.

[8] KANNE, Elena Cortona. Engine thermomanagement for fuel consumption reduction. 2000. Tese de Doutorado. ETH Zurich.

[9] MELO, T. de; MACHADO, G.; CARVALHO, L. de Oliveira; BELCHIOR, C. et al. In Cylinder Pressure Curve and Combustion Parameters Variability with Ethanol Addition. SAE Technical Paper. 2012.

[10] SIMONS, Michael Richard. Modellbildung und Parameteridentifikation für die Wandfilmdynamik eines Otto-Motors. Tese de Doutorado. ETH Zurich. 2001. 